\title{
Ação da levodopa e sua influência na voz e na fala de indivíduos com doença de Parkinson
}

\section{The action of levodopa and its influence on voice and speech of patients with Parkinson desease}

\author{
Luciana Lemos de Azevedo ${ }^{1}$, Francisco Cardoso ${ }^{2}$
}

\begin{abstract}
RESUMO
A doença de Parkinson, doença degenerativa progressiva decorrente da morte de células da substância negra compacta e outros núcleos pigmentados do tronco encefálico, é caracterizada por um esgotamento seletivo do neurotransmissor dopamina. Desta forma, acomete principalmente o sistema motor e acaba por se refletir em alterações comunicativas. A levodopa, que é convertida em dopamina, é o recurso farmacológico mais eficaz para o seu tratamento. A literatura mostra, através de alguns estudos, que a levodopa é capaz de proporcionar melhora em alguns parâmetros vocais, tais como aumento da frequência fundamental, da variação melódica, da intensidade vocal, da velocidade de fala, do tempo máximo de fonação e dos valores de pressão respiratória; melhora na inteligibilidade da fala, no tipo de voz; redução do grau do tremor vocal ou mesmo sua eliminação em alguns casos. No entanto, estudos mais recentes têm relatado que o efeito desta droga nos aspectos prosódicos da fala de parkinsonianos é bastante modesto, já que poucas variáveis prosódicas são modificadas após seu uso. Tem sido observado que a levodopa promove melhora nos parâmetros de duração e o tratamento fonoaudiológico e a associação dos tratamentos (fonoaudiológico e medicamentoso) promove a melhora de todos os parâmetros prosódicos: frequência, duração e intensidade, o que evidencia a importância da associação desses tratamentos, visando ao melhor desempenho comunicativo e benefícios motores globais que a levodopa proporciona.
\end{abstract}

Descritores: Doença de Parkinson/quimioterapia; Levodopa/uso terapêutico; Transtornos da articulação/terapia; Distúrbios da voz/ terapia; Fonoterapia

\section{INTRODUÇÃO}

A doença de Parkinson (DP), inicialmente descrita pelo médico inglês James Parkinson em 1817, é considerada uma doença degenerativa progressiva do sistema extrapiramidal, decorrente da morte de células da substância negra compacta e outros núcleos pigmentados do tronco encefálico, e que produz um esgotamento seletivo do neurotransmissor dopamina. Tal doença ocorre tipicamente por volta dos 50 aos 75 anos de idade, em ambos os $\operatorname{sexos}^{(1-2)}$.

A DP atinge $1 \%$ dos americanos acima de 50 anos de idade e vem somando 50 mil novos casos por ano ${ }^{(3)}$. Estima-se uma incidência de $1 / 400$ para a população como um todo e 1/200 para a população a partir de 40 anos de idade ${ }^{(4)}$, havendo pre-

(1) Doutora, Professora do Curso de Fonoaudiologia da Pontifícia Universidade Católica de Minas Gerais - PUC-Minas - Belo Horizonte (MG), Brasil.

(2) Professor Associado do Setor de Neurologia do Departamento de Clínica Médica da Faculdade de Medicina da Universidade Federal de Minas Gerais - UFMG - Belo Horizonte (MG), Brasil.

Endereço para correspondência: Luciana Lemos de Azevedo, R. Pio Porto de Menezes, 120/1401A, Bairro Luxemburgo, Belo Horizonte - MG, CEP: 30380-300. E-mail: azevedoll@terra.com.br

Recebido em: 26/2/2008; Aceito em: 4/10/2008 domínio para o sexo masculino ${ }^{(4-5)}$. Sua prevalência é estimada em cem a duzentos casos para cada cem mil pessoas ${ }^{(5)}$. Recente estudo brasileiro ${ }^{(6)}$ mostrou que $3,4 \%$ dos brasileiros acima de 64 anos de idade têm DP.

O diagnóstico da DP, essencialmente clínico, baseia-se nos dados coletados na anamnese e no exame físico. As manifestações do parkinsonismo se caracterizam por sinais e sintomas basicamente motores: bradicinesia associada a pelo menos um outro sinal cardeal de parkinsonismo (rigidez muscular, tremor ou alteração de reflexos posturais). Tais sinais acabam por influenciar a produção de fala e, ainda, por acarretar uma expressão facial em "máscara" (hipomimia), interferindo de forma negativa na expressão comunicativa e na qualidade de vida desses indivíduos ${ }^{(2,4)}$. Observam-se ainda, outros sinais, tais como micrografia (caligrafia muito pequena) e dores musculares ${ }^{(4,7)}$.

Além do prejuízo na função comunicativa, a DP também acarreta disfagia. Porém, mesmo com alterações bastante marcantes na deglutição, os parkinsonianos só costumam apresentar queixas referentes à deglutição em estágios mais avançados da doença. Um estudo que ilustra este fato ${ }^{(8)}$, evidenciou que os indivíduos com DP estudados, mesmo sem queixas de deglutição, apresentavam disfagia. Tal falta de 
correlação entre a presença da queixa e da disfagia, provavelmente se dá em função de uma redução na sensibilidade do trato digestivo. Considerando a evolução da doença, alguns autores ${ }^{(9)}$ observaram alterações de deglutição proporcionais ao estágio evolutivo da DP. Porém, tais autores observaram a presença de queixas consistentes de deglutição apenas em estágios mais avançados da doença, mesmo na presença de alterações significativas em estágios iniciais.

A progressão da doença é extremamente variável entre pacientes e, aqueles que manifestam o tremor como sintoma inicial, costumam apresentar um prognóstico mais favorável ${ }^{(1)}$. O início da doença em idade avançada pode estar associado à rápida progressão e ocorrência de danos cognitivos. A doença reduz a expectativa de vida, que pode ser, pelo menos em parte, restaurada pelo tratamento com levodopa e outras drogas.

A levodopa, que é convertida em dopamina, corrige o problema bioquímico da DP. Não há dúvidas quanto à sua efetividade na melhora dos sintomas motores globais causados pela doença. O mesmo não acontece em relação ao efeito da levodopa na voz e fala de parkinsonianos, sendo que vários estudos têm abordado a interferência da administração da levodopa no desempenho comunicativo dos parkinsonianos.

\section{REVISÃO DA LITERATURA}

Assim como referido na literatura, na prática clínica observamos que pacientes com DP apresentam uma incidência significativa de transtornos na comunicação oral, o que se dá em função dos sintomas motores resultantes da referida doença. Estima-se que $89 \%$ dos pacientes com DP experimentarão alterações vocais com a progressão da doença ${ }^{(10)}$, ao passo que 70 a $85 \%$ dos pacientes com DP apresentam alterações na fala $^{(11)}$, em decorrência dos sintomas motores presentes nesta doença.

O prejuízo peculiar da expressão verbal observado no paciente parkinsoniano caracteriza-se por monotonia de frequência e pouca variação na intensidade ${ }^{(4,12-16)}$; loudness reduzida $^{(15-16)}$; qualidade vocal rouca-áspera-soprosa, tremor vocal, insuficiência prosódica ${ }^{(2)} ;$ imprecisão articulatóriaa ${ }^{(2,7,16)}$; disfluência ${ }^{(17)}$; alteração da velocidade de fala ${ }^{(7,10-11,15-16,18)}$ e pequenos jatos de fala com pausas inadequadas ${ }^{(10-11,15,18)}$; repetição de palavras ou sílabas ${ }^{(4)}$ e ritmo alterado ${ }^{(7)}$. Estas alterações podem estar presentes em fases precoces da enfermidade ${ }^{(18)}$ e aumentar sua intensidade e frequência de ocorrência com a duração e evolução da doença ${ }^{(18)}$.

Além do prejuízo na função comunicativa, a DP também pode levar à disfagia, hipomimia, micrografia, alterações na marcha, depressão, alterações autonômicas, e respiratórias. A disfunção respiratória é a principal causa de morte de pacientes com DP, sendo a pneumonia por aspiração o problema mais comum $^{(19)}$. Vale lembrar que, apesar de a DP ser considerada uma doença característica de transtorno do movimento, algumas características não-motoras são típicas da doença, como alterações cognitivas, distúrbios do sono e distúrbios sensoriais $^{(20)}$.

Apesar de a levodopa ser a medicação mais importante no tratamento da DP, outras drogas também podem ser utilizadas: as neuroprotetoras (que visam atrasar a progressão da degeneração neuronal - efeito não provado para qualquer medicação) e as drogas que visam potencializar a ação da levodopa (agonistas dopaminérgicos e inibidores de COMT - catecol-O-metiltransferase). Os inibidores de COMT atuam uniformizando o perfil de tempo de concentração plasmática da levodopa e estendendo sua distribuição no cérebro, uma vez que a variação na distribuição da levodopa é um fator crítico no desenvolvimento de flutuações da resposta. Com isto, os inibidores de COMT melhoram a ação da levodopa, reduzindo o extenso metabolismo pelo qual esta passa e causando uma variação extensa na sua concentração plasmática ${ }^{(21-24)}$.

Os agonistas dopaminérgicos, por sua vez, são drogas que estimulam diretamente os receptores dopaminérgicos e têm sido desenvolvidos na tentativa de superar as limitações terapêuticas da levodopa. Os agonistas dopaminérgicos têm meia vida mais longa que a levodopa, possibilitando uma estimulação mais prolongada (mais contínua) dos núcleos da base, o que não ocorre com a levodopa, que produz uma estimulação pulsátil, que levaria às complicações. Dessa maneira, há redução na tendência a complicações geradas pela administração a longo prazo da levodopa, cuja causa é a estimulação intermitente dos receptores dopaminérgicos.

O tratamento exclusivamente com agonistas dopaminérgicos tende a reduzir o índice de desenvolvimento de complicações motoras; porém, com a evolução da doença, este medicamento não é suficiente para produzir benefícios clínicos satisfatórios e torna-se inevitável o uso da levodopa ${ }^{(23-24)}$. Um aspecto negativo importante deste tipo de droga é a tendência ao desenvolvimento de distúrbios psiquiátricos, especialmente em pacientes acima de $70 \operatorname{anos}^{(25)}$. Além disto, são claramente menos eficazes do que a levodopa em reverter as manifestações do parkinsonismo. Em contrapartida, a levodopa seria a primeira escolha em pacientes com grande incapacidade motora e prejuízo dos reflexos posturais e pacientes idosos acima de $70 \operatorname{anos}^{(5)}$.

A introdução da levodopa no início da década de 60 revolucionou o tratamento sintomático da DP. A literatura é unânime em admitir que a levodopa é o recurso farmacológico mais eficaz para o tratamento da DP idiopática (DPI) ${ }^{(25)}$. Em tese, todos os pacientes com DP serão tratados com a levodopa, que é convertida em dopamina ${ }^{(21,25-26)}$.

Além de melhorar os sintomas da DP, a levodopa está associada a um decréscimo na taxa de mortalidade dessa população ${ }^{(4,23)}$. Uma boa resposta ao uso da levodopa nos primeiros anos da evolução da DP é um sinal de extrema importância no diagnóstico diferencial entre a DPI e outras síndromes parkinsonianas, tais como atrofia de múltiplos sistemas, paralisia supranuclear progressiva e parkinsonismo secundário ${ }^{(22)}$.

A levodopa é convertida em dopamina e corrige, teoricamente, o defeito bioquímico da DP. Ela é absorvida no trato gastrointestinal e distribuída para outros tecidos, principalmente músculos, sendo que uma dieta rica em proteínas pode interferir negativamente na passagem da levodopa para o sangue ${ }^{(23)}$. A velocidade do esvaziamento gástrico é um fator que interfere no transporte da levodopa para o sistema nervoso central. Outro obstáculo que a levodopa deve vencer é a barreira hematoencefálica (da corrente sanguínea para o cérebro). No início da doença, como os neurônios residuais 
da substância negra armazenam dopamina, esses fatores não costumam prejudicar a resposta à levodopa. Porém, com a evolução da doença e consequente perda progressiva de neurônios, o sistema nervoso passa a ser totalmente dependente da dopamina proveniente da medicação. A partir deste momento, irregularidades no esvaziamento gástrico e/ou dieta rica em proteína, que compete com a levodopa no trato gastrointestinal e na barreira hematoencefálica, resultam em uma maior latência entre a tomada da medicação e o início de seu efeito ${ }^{(5,21,23,25)}$. Dessa forma, é importante que o paciente tenha o cuidado de administrar a levodopa pelo menos uma hora após as refeições e deixar os alimentos com alto teor de proteína para a última refeição do dia ${ }^{(21)}$. Ou, ainda, fazer uso da levodopa com o estômago vazio ou junto com uma dieta rica em carboidratos - os quais facilitarão sua absorção intestinal, e desviar a dieta protéica para, pelo menos, duas horas após a administração da levodopa ou ao final do dia ${ }^{(25)}$.

Apesar de a meia vida da levodopa ser de apenas 60 a 90 minutos ${ }^{(23)}$, normalmente, no início do uso da medicação, sua ação estende-se por um maior número de horas na grande maioria dos pacientes, sendo que, em pacientes com formas leves de DP, a ação da levodopa pode durar até oito ou 12 horas $^{(25)}$. No entanto, com o passar do tempo, a duração do efeito da medicação começa a reduzir-se, e o paciente começa a perceber e distinguir claramente os momentos em que seu desempenho funcional é satisfatório, devido ao efeito da medicação ("período ligado"; ou período on) e os momentos em que o desempenho funcional é inferior, devido à interrupção do efeito da levodopa ("período desligado"; ou período off). Estas variações no desempenho funcional são chamadas de flutuações e são consideradas complicações do uso da levo$\operatorname{dopa}^{(23,25)}$.

Cerca de 40 anos após sua introdução no mercado, a levodopa continua sendo a medicação mais efetiva no tratamento sintomático da $\mathrm{DP}^{(27-28)}$. Porém, depois de um período de resposta satisfatória à levodopa, os pacientes normalmente começam a apresentar complicações em decorrência deste tratamento medicamentoso ${ }^{(25)}$. Acredita-se que tais complicações atinjam $50 \%$ dos parkinsonianos com cinco anos de uso da levodopa ${ }^{(29)}$.

Provavelmente a questão central no que se refere às complicações decorrentes do uso prolongado da levodopa é a estimulação intermitente dos receptores dopaminérgicos, em função da perda da capacidade de armazenamento de dopamina, devido à morte de neurônios da substância negra, o que expõe os receptores dopaminérgicos a concentrações de dopamina alternadamente altas e baixas ${ }^{(21-25)}$.

Dentre os fatores que podem contribuir para o encurtamento da duração do efeito da levodopa, temos: a lentidão do esvaziamento gástrico, a irregularidade na absorção da levodopa no trato gastrointestinal e na sua passagem na barreira hematoencefálica, o desaparecimento da capacidade de armazenamento da levodopa devido a uma acentuada perda de neurônios da parte compacta da substância negra e, principalmente, fatores farmacodinâmicos, ou seja, aos mecanismos cerebrais de atuação da medicação ${ }^{(21,25)}$.

A resposta terapêutica à levodopa apresenta dois componentes: a resposta de curta duração (na qual se vê uma melhora da incapacidade motora em minutos ou horas, enquanto o plasma sanguíneo possui altos níveis de concentração da medicação, após a administração desta) e a resposta de longa duração (em que o benefício motor é preservado por horas ou dias após a interrupção da administração da medicação) $)^{(27)}$. A interrupção da administração da medicação pelo período de doze horas não traz riscos nem prejuízos importantes para os parkinsonianos, uma vez que os efeitos de longa duração permanecem ${ }^{(27)}$. Por isso, a interrupção do uso da medicação por este período tem sido utilizada em pesquisas que visam à avaliação do indivíduo com DP no estado off. Experimentalmente, esta situação é chamada de "estado off praticamente definido"(30).

As flutuações do efeito terapêutico da levodopa podem ocorrer de diversas formas, mas temos dois tipos mais comuns. Um deles é a deterioração de fim de dose ("wearing off'), que é caracterizada pela perda gradual do efeito benéfico da levodopa, que pode se reduzir para uma a duas horas. O outro tipo de flutuação mais comum, chamado de "ligamento-desligamento" ("on-off'), acontece quando a perda do efeito terapêutico da levodopa se dá de forma abrupta; o que pode durar de alguns minutos a horas. Essa perda é seguida de um retorno, também abrupto, do efeito benéfico da levodopa, sem que tenha havido ingestão de nova dose da droga ${ }^{(22)}$.

A idade do paciente é um dos fatores que influencia a ocorrência de flutuações e discinesias, sendo que indivíduos mais jovens tendem a ter maior predisposição ao desenvolvimento de tais complicações ${ }^{(25)}$. Além da idade, há outros fatores de risco que podem contribuir para o surgimento de complicações, como a duração da doença, a dosagem de levodopa administrada, a duração do tratamento com esta medicação ${ }^{(28)}$, bem como a gravidade da doença ${ }^{(31)}$. Dessa forma, normalmente é aconselhado que se adie o máximo possível o início do tratamento com a levodopa ${ }^{(21-26,28)}$.

Outra complicação que pode ser observada devido ao uso da levodopa são as discinesias (movimentos anormais involuntários) que, na grande maioria das vezes, ocorrem em associação com o ciclo de flutuações do efeito da levodopa ${ }^{(24-26,31)}$. A intensidade das discinesias varia muito de um indivíduo para outro, e aquelas que causam impacto funcional significativo ocorrem em cerca de $30 \%$ dos parkinsonianos que fazem uso da levodopa ${ }^{(25)}$. A literatura ${ }^{(24)}$ relata que as discinesias decorrem da associação de dois fatores: a intensidade do prejuízo dopaminérgico e a administração crônica da levodopa. Há uma prevalência de discinesia de $15 \%$ a $80 \%$ após cinco anos de tratamento com a levodopa ${ }^{(23)}$. Outros autores ${ }^{(32)}$ verificaram, a partir de uma revisão da literatura, que a frequência média de ocorrência de discinesias e flutuações motoras é de aproximadamente $40 \%$, após quatro a seis anos de uso da levodopa. Vale lembrar que o tratamento das complicações motoras, tais como flutuações e discinesias, não é tarefa fácil, e envolve uma série de procedimentos.

Além das manifestações motoras, outros problemas, tais como disfunção do controle do sistema nervoso autônomo (por exemplo, crises de sudorese, distúrbios de esvaziamento gástrico, distúrbios do apetite, distúrbios de salivação, etc.), disfagia, problemas sensoriais (como dor, dormência) e psiquiátricos (como depressão, distúrbios do sono, alucinações), 
também são sintomas das flutuações nos pacientes em uso de levodopa ${ }^{(25)}$.

Quanto à influência da levodopa nos parâmetros de voz e de fala, observamos, na literatura, estudos que ilustram efeitos positivos, bem como efeitos modestos ou até mesmo negativos. Em relação aos efeitos positivos, alguns estudos referiram que a levodopa leva a um aumento da frequência fundamental $\left(\mathrm{F}_{0}\right)$ ${ }^{(12)}$, da variação melódica ${ }^{(12)}$ e da intensidade vocal ${ }^{(33)}$, além de melhora na inteligibilidade da fala, no tipo de $\mathrm{voz}^{(34)}$ e na velocidade de fala, que se torna mais rápida ${ }^{(12)}$.

Autores ${ }^{(35)}$ observaram, através de um estudo cujo objetivo era verificar o efeito da levodopa sobre a respiração e a fonação de indivíduos com DPI, aumento significativo no tempo máximo de fonação após a administração da medicação, o que refletiu a influência positiva da levodopa no controle do fluxo aéreo durante a fonação, apesar de não ter sido observado aumento significativo do volume respiratório e da intensidade vocal. Outros autores ${ }^{(36)}$ também observaram uma melhora estatisticamente significativa no tempo máximo de fonação em função da administração da levodopa. Outro achado relatado por estes mesmos autores, em outro estudo ${ }^{(37)}$, foi a eficácia da levodopa na redução do grau do tremor vocal de indivíduos com DPI e na eliminação desse tremor em alguns casos.

Um estudo ${ }^{(38)}$ que analisou o padrão respiratório e as pressões respiratórias máximas de indivíduos com DP antes e após a administração da levodopa, verificou que tal medicação influencia de maneira positiva (apesar de modestamente) as pressões respiratórias máximas de parkinsonianos entre as escalas 2 e 3 de Hoehn \& Yahr, tendo sido observado um aumento significativo nos valores de pressão respiratória máxima após a administração da levodopa.

No entanto, alguns estudos ${ }^{(12-14)}$ observaram que o efeito desta droga nos aspectos prosódicos da fala de parkinsonianos é bastante modesto, já que poucas variáveis prosódicas foram modificadas após seu uso. Isto indica que outras medidas terapêuticas, como terapia da fala, podem desempenhar papel importante no tratamento dos transtornos de fala em indivíduos com DP.

Por outro lado, em outro estudo ${ }^{(39)}$ foi possível observar melhora nos aspectos prosódicos da fala após a administração da levodopa; porém, apenas para o parâmetro prosódico $\mathrm{F}_{0}$ (média e desvio padrão), quando avaliaram a repercussão do tratamento com a levodopa e/ou com a estimulação eletrofisiológica dos núcleos subtalâmicos nos três parâmetros prosódicos $\left(\mathrm{F}_{0}\right.$, duração e intensidade) em um grupo de 20 pacientes parkinsonianos (dez submetidos a cada tipo de tratamento).

Mais recentemente, um estudo ${ }^{(40)}$ verificou que a DP prejudica a produção eficiente dos parâmetros prosódicos (tendo como referência um grupo controle de idosos), sendo que após a administração da levodopa, foi observada uma melhora significativa nos parâmetros de duração; mas, mesmo assim, os parkinsonianos não chegaram a ter um desempenho tão satisfatório quanto o grupo controle. O tratamento medicamentoso (através da levodopa) promoveu melhora nos parâmetros de duração e o tratamento fonoaudiológico (através de uma adaptação do Método Lee Silverman de Tratamento Vocal para duas sessões semanais, mantendo o total de 16 sessões) e a associação dos tratamentos (fonoaudioló- gico e medicamentoso) promoveram a melhora de todos os parâmetros prosódicos: frequência, duração e intensidade. $\mathrm{O}$ tratamento fonoaudiológico teve o diferencial de apresentar mais benefícios para a variável frequência (aumentando, além das taxas de mudança de variação melódica da tônica nuclear e átona pretônica, a tessitura do enunciado e a amplitude de variação melódica da tônica nuclear e da átona pretônica). Isso se deve, provavelmente, ao fato de os déficits de fala na DP relacionados aos parâmetros prosódicos frequência e intensidade, não serem resultado de alterações dopaminérgicas e, desta forma, não serem influenciados pela administração da levodopa, ao contrário do que acontece com o parâmetro duração. De qualquer forma, o ideal é que sejam associados os tratamentos fonoaudiológico e medicamentoso, tendo em vista que ambos favorecem o desempenho comunicativo, além dos benefícios motores globais que a levodopa proporciona, e a melhora na qualidade de vida que ambos os tratamentos, consequentemente, promovem.

\section{DISCUSSÃO}

A análise dos dados da literatura nos permitiu verificar que apesar dos possíveis efeitos colaterais, a levodopa é o recurso farmacológico mais eficaz para o tratamento sintomático da $\mathrm{DPI}^{(25,27-28)}$, sendo que, a princípio, todos os pacientes com DP serão tratados com esta droga ${ }^{(21,25-26)}$. A levodopa, além de melhorar os sintomas da DP, está associada a um decréscimo na taxa de mortalidade dessa população ${ }^{(4,23)}$ e é de grande importância para o diagnóstico diferencial entre a DP e outras síndromes parkinsonianas, que não apresentam boa resposta ao uso da levodopa, mesmo nos primeiros anos da evolução da doença ${ }^{(22)}$.

No que se refere à influência da levodopa nos parâmetros de voz e fala, a literatura aponta vários benefícios desta medicação, que, após sua administração proporciona ao parkinsoniano aumento da $\mathrm{F}_{0}^{(12)}$, da variação melódica ${ }^{(12)}$, da intensidade vocal $^{(33)}$, da velocidade de fala ${ }^{(12)}$, do tempo máximo de fonação ${ }^{(35)}$ e dos valores de pressão respiratória máxima ${ }^{(38)}$. Tal medicação proporciona, ainda, melhora na inteligibilidade da fala, no tipo de $\mathrm{voz}^{(34)}$, bem como é capaz de reduzir o grau do tremor vocal de indivíduos com DPI, ou, em alguns casos, eliminar esse tremor ${ }^{(37)}$.

Porém, quando abordado mais especificamente os parâmetros prosódicos da fala, a literatura mostra que a levodopa não é tão eficiente, podendo interferir apenas na melhora de um dos parâmetros. Um estudo ${ }^{(39)}$ evidenciou melhora nos aspectos prosódicos da fala após a administração da levodopa; porém, apenas para o parâmetro prosódico $\mathrm{F}_{0}$. Já outro estudo mais recente, verificou que após a administração da levodopa, é possível observar melhora significativa nos parâmetros de duração; mas, mesmo assim, os parkinsonianos não chegaram a ter um desempenho tão satisfatório quanto o grupo contro$\mathrm{l}^{(40)}$. Essa mesma autora observou que a levodopa promoveu melhora nos parâmetros de duração e o tratamento fonoaudiológico e a associação de ambos os tratamentos promoveram a melhora de todos os parâmetros prosódicos: frequência, duração e intensidade, o que reforça a validade da associação dos tratamentos fonoaudiológico e medicamentoso na melhora do desempenho comunicativo do indivíduo com DP. 


\section{COMENTÁRIOS FINAIS}

A vivência clínica nos permite observar que apesar de a levodopa apresentar importante impacto positivo nos sintomas motores globais da DP, o mesmo não é observado para a expressão oral nessa enfermidade.

A presente revisão de literatura apontou para uma possível explicação para esta realidade: provavelmente, o parâmetro prosódico duração apresenta evidente melhora após a administração da levodopa, tendo em vista que ela interfere diretamente nesta variável, o que também pode ser observado nos sintomas motores globais, quando verificamos melhora do sintoma bradicinesia após administração da levodopa. Por outro lado, o fato de os déficits de fala na DP relacionados aos parâmetros prosódicos frequência e intensidade não serem resultado de alterações dopaminérgicas, faz com que esses não sofram interferência do uso da medicação ${ }^{(40)}$.

O fato de o efeito da levodopa nos aspectos prosódicos da fala de parkinsonianos ter se mostrado bastante modesto ${ }^{(12-14,40)}$, reforça a necessidade da associação de outras medidas terapêuticas, como terapia da fala, visando o tratamento dos transtornos de fala em indivíduos com DP. Dessa forma, o ideal é que sejam associados os tratamentos fonoaudiológico e medicamentoso, o que promoveria benefícios motores globais por meio da administração da levodopa, bem como benefícios no desempenho comunicativo, por meio da fonoterapia, o que, consequentemente, promove melhora na qualidade de vida dos indivíduos com DP.

\begin{abstract}
Parkinson's disease (PD), a progressive degenerative disease characterized by death of neurons of pars compacta of substantia nigra and other pigmented nucleii of the brainstem, is associated with dopamine depletion. Clinically, it is characterized primarily by motor deficits which may interfere with communication. Levodopa, which is converted into dopamine, is the most effective drug for the treatment of PD. A myriad of studies show that levodopa improves several vocal paremeters, resulting in increase of fundamental frequency, melodic variation, vocal intensity, speed of the speech, maximum speech time and values of respiratory pressure. Moreover, it improves speech intelligibility and type of voice, as well as reduces or even suppresses vocal tremor. However, more recent data have shown that the effect of this drug on prosodic aspects of parkinsonian speech is quite modest, since few prosodic variables are modified after its use. It has been observed that levodopa improves the duration parameters of speech, whereas speech therapy, alone and combined with the use of levodopa, results in improvements on all prosodic parameters: frequency, duration and intensity. This highlights the importance of the association of these treatments for better communication and global motor benefits.
\end{abstract}

Keywords: Parkinson disease/drug therapy; Levodopa/therapeutic use; Articulation disorders/therapy; Voice disorders/therapy; Speech therapy

\title{
REFERÊNCIAS
}

1. Hoehn MM, Yahr MD. Parkinsonism: onset, progression and mortality. Neurology. 1967;17(5):427-42.

2. Silveira DN, Brasolotto AG. Reabilitação vocal em pacientes com doença de Parkinson: fatores interferentes. Pró-Fono. 2005;17(2):24150.

3. Vitorino MR, Homem FCB. Doença de Parkinson: da fonação à articulação. Fono Atual. 2001;4(17):35-9.

4. André ES. Moléstia de Parkinson. Fisioter Mov. 2004;17(1):11-24.

5. Teixeira Júnior AL, Cardoso F. Tratamento inicial da doença de Parkinson. Neurociências. 2004;12(3):141-6.

6. Barbosa MT, Caramelli P, Maia DP, Cunningham MC, Guerra HL, Lima-Costa MF, Cardoso F. Parkinsonism and Parkinson's disease in the elderly: a community-based survey in Brazil (the Bambuí study). Mov Disord. 2006;21(6):800-8.

7. Jorge TM, Lamônica DAC, Caldana ML. Doença de Parkinson: transtornos da comunicação oral e gráfica. Fono Atual. 2004;7(30):1425.

8. Azevedo LL, Cardoso F, Marques P, Oliveira DV, Martins AJM. Doença de Parkinson idiopática: queixa de disfagia $\mathrm{X}$ achados ao exame nasoendoscópico. In: XII Congresso Brasileiro de Fonoaudiologia e II Congresso Sul Brasileiro de Fonoaudiologia; 2004 Out 6-9; Foz do Iguaçu. Anais. Rev Soc Bras Fonoaudiol. 2004; Supl Esp.

9. Gozzoni J, Pedroso KCDA, Grolli EB. Avaliação fonoaudiológica funcional em pacientes portadores de doença de Parkinson. Rev CEFAC. 2003;5:223-6.
10. Perez KS, Ramig LO, Smith ME, Dromey C. The Parkinson larynx: tremor and videostroboscopic findings. J Voice. 1996;10(4):354-61.

11. De Angelis EC. Doença de Parkinson: efetividade da fonoterapia na comunicação oral e na deglutição [tese]. São Paulo: Universidade Federal de São Paulo. Escola Paulista de Medicina; 1995.

12. Azevedo LL. Aspectos prosódicos da fala do parkinsoniano [dissertação]. Belo Horizonte: Universidade Federal de Minas Gerais. Faculdade de Letras; 2001.

13. Azevedo LL, Cardoso F, Reis C. Análise acústica da prosódia em mulheres com doença de Parkinson: efeito da levodopa. Arq Neuropsiquiatr. 2003;61(4):995-8.

14. Azevedo LL, Cardoso F, Reis C. Análise acústica da prosódia em mulheres com doença de Parkinson: comparação com controles normais. Arq Neuropsiquiatr. 2003;61(4):999-1003.

15. Mourão LF. Avaliação da fonoarticulação de pacientes com doença de Parkinson pré e pós-palidotomia [tese]. São Paulo: Universidade Federal de São Paulo. Escola Paulista de Medicina; 2002.

16. Paula Soares MF, Albano EC. Dinâmica da produção da fala em sujeitos portadores de Doença de Parkinson: análise das vogais. In: IX Congresso Nacional e III Congresso Internacional de Fonética e Fonologia; 2006 Nov 27-29; Belo Horizonte. Caderno de Resumos. Belo Horizonte: UFMG, 2006. v. 1. p. 13.

17. Goberman AM, Blomgren M. Parkinsonian speech disfluencies: effects of L-dopa-related fluctuations. J Fluency Disord. 2003;28(1):55-70.

18. Pawlas AA, Ramig LO, Countryman S. Perceptual voice and speech 
characteristics in patients with idiopathic Parkinson. NCVS Status and Progress Report. 1996;10:79-87.

19. Onodera H, Okabe S, Kikuchi Y, Tsuda T, Itoyama Y. Impaired chemosensitivity and perception of dyspnoea in Parkinson's disease. Lancet. 2000;356(9231):739-40. Comment in: Lancet. 2000;356(9247):2099. Lancet. 2000;356(9247):2100.

20. Samii A, Nutt JG, Ransom BR. Parkinson's disease. Lancet. 2004;363(9423):1783-93. Review.

21. Andrade LAF. Estratégias no tratamento do paciente jovem. In: Andrade LAF, Barbosa ER, Cardoso F, Teive HAG. Doença de Parkinson: estratégias atuais de tratamento. São Paulo: Lemos Editorial; 1999. p. 101-31.

22. Jankovic J. Complications and limitations of drug therapy for Parkinson's disease. Neurology. 2000;55(12 Suppl 6):S2-6.

23. Koller WC. Levodopa in the treatment of Parkinson's disease. Neurology. 2000;55(11 Suppl 4):S2-7; discussion S8-12.

24. Olanow CW, Obeso JA. Pulsatile stimulation of dopamine receptors and levodopa-induced motor complications in Parkinson's disease: implications for the early use of COMT inhibitors. Neurology. 2000;55(11 Suppl 4):S72-7; discussion S78-81.

25. Cardoso F. Fisiopatologia das flutuações e discinesias induzidas por levodopa na doença de Parkinson. In: Andrade LAF, Barbosa ER, Cardoso F, Teive HAG. Doença de Parkinson: estratégias atuais de tratamento. São Paulo: Lemos Editorial; 1999. p. 21-32 .

26. Katzenschlager R, Lees AJ. Treatment of Parkinson's disease: levodopa as the first choice. J Neurol. 2002;249 Suppl 2:II19-24.

27. Zappia M, Oliveri RL, Montesanti R, Rizzo M, Bosco D, Plastino M, et al. Loss of long-duration response to levodopa over time in PD: implications for wearing-off. Neurology. 1999;52(4):763-7. Comment in: Neurology. 2002;58(5):837; author reply 837-8.

28. Kostic VS, Marinkovic J, Svetel M, Stefanova E, Przedborski S. The effect of stage of Parkinson's disease at the onset of levodopa therapy on development of motor complications. Eur J Neurol. 2002;9(1):9-14.

29. Schrag A, Jahanshahi M, Quinn N. What contributes to quality of life in patients with Parkinson's disease? J Neurol Neurosug Psychiatry. 2000;69(3):308-12.

30. Langston JW, Widner H, Goetz CG, Brooks D, Fahn S, Freeman T, Watts R. Core assessment program for intracerebral transplantations (CAPIT). Mov Disord. 1992;7(1):2-13.
31. Vaamonde J, Ibáñez R, Gudín M, Hernández A. [Fluctuations and dyskinesias as early L-dopa-induced motor complications in severe Parkinsonian's patients]. Neurologia. 2003;18(3):162-5. Spanish.

32. Ahlskog JE, Muenter MD. Frequency of levodopa-related dyskinesias and motor fluctuations as estimated from the cumulative literature. Mov Disord. 2001;16(3):448-58.

33. Jiang J, Lin E, Wang J, Hanson DG. Glottographic measures before and after levodopa treatment in Parkinson's disease. Laryngoscope. 1999;109(8):1287-94.

34. Mourão LF. Estudo de comunicação oral em pacientes com doença de Parkinson em fase: "off" e "on", com o tratamento pela levodopa [tese]. São Paulo: Universidade Federal de São Paulo. Escola Paulista de Medicina; 1997.

35. Guedes LU, Azevedo LL, Cardoso F, Parreira VF, Reis C. Efeito da levodopa sobre a respiração e fonação dos indivíduos com doença de Parkinson idiopática. In: XIII Congresso Brasileiro de Fonoaudiologia, 2005, Santos. Anais. Rev Soc Bras Fonoaudiol. 2005; Supl Esp.

36. Azevedo LL, Guedes LU, Cardoso F, Reis C, Parreira VF. Correlação da influência da levodopa sobre os aspectos motores, respiratórios e fonatórios dos indivíduos com doença de Parkinson. In: XIII Congresso Brasileiro de Fonoaudiologia, 2005, Santos. Anais. Rev Soc Bras Fonoaudiol. 2005; Supl Esp.

37. Azevedo LL, Vieira RM, Cardoso F, Reis C. Efeito da levodopa no tremor vocal de pacientes parkinsonianos. In: XIII Congresso Brasileiro de Fonoaudiologia, 2005, Santos. Anais. Rev Soc Bras Fonoaudiol. 2005; Supl Esp.

38. Guedes LU. Padrão respiratório e pressões respiratórias máximas em indivíduos com doença de Parkinson sem o efeito e sob o efeito da levodopa em comparação a indivíduos assintomáticos [dissertação]. Belo Horizonte: Universidade Federal de Minas Gerais. Escola de Educação Física, Fisioterapia e Terapia Ocupacional; 2005.

39. Viallet F, Teston B, Jankowski L, Purson A, Peragut JC, Regis J, Witjas T. Speech Prosody Conference. Effects of pharmacological versus electrophysiological treatments on parkinsonian dysprosody [Internet]. [cidted 2008 Apr 12]. Available from: http://www.lpl.univ-aix.fr/sp2002/ papers.htm

40. Azevedo LL. Expressão da atitude através da prosódia em indivíduos com doença de Parkinson idiopática [tese]. Belo Horizonte: Universidade Federal de Minas Gerais. Faculdade de Letras; 2007. 